\title{
Septicemia neonatal: incidencia, letalidad y características bacteriológicas. Comparación de dos períodos
}

\author{
Marcela Concha R. '; Marta Azócar P.'; Carolina Letelier B.'; \\ Ada Chicharro C. '; María Isabel Saldías R. ${ }^{2}$; José Luis Tapia . $^{3}$
}

\begin{abstract}
Resumen
Objefivos: Jescribir y comparar la incidencia y letalidad de lo septicemia neonatal, en dos periodos en un hospital universitario. Pocientes y méílodo: se compararon recién nacidos que tuvieron septicemio entre los meses de julio de 1987 a junio de 1989 con los del periodo de eneio de 1995 a diciembre de 1996. Resultodos: lo incidencia de septicerria neonarol aumentó $\{2,9$ a 8,7 por mill y la leralidad $[44 \%$ a $2,2 \%\}$ disminuyó en el segundo periodo. Los poblaciones esludiadas y los mélodos de diagnóstico fueron similores. En las sepricemias connatoles el germen preponderanle fue el Sireptococcus grupo 3 sin diferencias entre períodos (50\% ante $52 \%$ ). Entre las seplicemias nosocomiales, aumentcron las praducidas por estafilococo [desde 25\% o 91\%], siendo Staphylococcus epidermidis devino el principal agenle durante el segundo periodo $157 \%$ del relall, disminuyendo la sensibilidad a cloxacilina (100\% a $33 \%$ ). Conclusiones: se demuestra un aumento de la incidencio y mejoría del pronóstico de las seplicernias neonalales cen un combio en sus caracteristicas tocteriológicos entre los períodos estudiados, similar a lo observado en atros paises.
\end{abstract}

(Palabras clave: recién nacidos, septıcernia.)

\section{Neonatal septicaemia: incidence, case-fatality and microbiological findings along two different periods}

Objectrve: lo desaibe frequency and casefatolity rale of neonalal septicaemia is the same medical center along wwo different periods and to analyse microbiological aspects of bot early and lole onset septicaemia. Potients and meshco's: al newborn infants wilh septicaemia identified olong a two year period from july 1987 throughout june 1989 were compared to cll those derected in a similar tergth period extending from january 1995 throughoul december 1996. Resuls: even though population and diagrostic criterio were similar, incidence of neonatal septicaemia increosed from 2.9 to 8.7 per thousand and cose-olality rale decreased from $44 \% 102.2 \%$ belween the first and the second periods. The most frequently isolated bocteria in early onsel septicaemia wos group B Streptococcus in both periods $150 \%$ versus $52 \%$ Frequency of slaphylococcal isolates increased in late onsel septicaemiaiffom $25 \%$ to $91 \%]$. Srophyloccccus epidermidis was the most common pothogen $(57 \%$ of totol) along 1 he second period, and their sensibility to cloxocilin decreosed from $100 \%$ to $33 \%$. Conclusion: increase in the incidence, prognosis improvemenl and a change in the bocteriolegy of neonatcl septicaemio between the study periods, are similar to those reported in other countries.

(Key words: newborn, septicoemia.)

Las infecciones bacterianas son causa frecuente de morbimortalidad en el período neona-

1. Internas. Facultad de Medicina, Pontificia Universidad Carolica de Chile.

2. Enfermera. Unidad de Neonatología. Departamento de Pediatría. Universidad Católica de Chile.

3. Departamento de Pediatría. Faculiad de Medicina. Pontificia Universidad Católica de Chile. tal, de ahí su importancia. Los mecanismos de inmunidad de los recién nacidos son relativamente deficientes en comparación con los de niños mayores, especialmente de los recién nacidos premaluros, que son más vulnerables a infectarse ${ }^{1-4}$. El origen de las infecciones neonatales puede estar en la madre (prenatal), en el parto (connatal) o en la hospitalización, 
por procedimientos $y$ terapias invasivas (nosocomiales). La incidencia de septicemia en recién nacidos varía de 2 a 10 por mil nacidos vivos $2,3,5.7$ y la letalidad es muy variables, entre menos de $10 \%$ y hasta $69 \%$ 1.2.5.7. Los agentes causales de las septicemias neonatales ha variado con el tiempo ${ }^{5-7}$.

La relevancia de este problema exige una vigilancia clínica y epidemiológica permanente. El objetivo primario de este estudio fue describir y comparar la incidencia y la letalidad de las septicemias en recién nacidos en una unidad de cuidados intensivos neonatales a lo largo de dos períodos. Secundariamente se analizaron las características poblacionales y bacteriologicas en ambos períodos comparando las sepsis connatales con las nosocomiales.

\section{Pacientes y método}

Se incluyeron todos los recién nacidos hospitalizados en la unidad de cuidado iniensivo neonatal del Hospital Clínico de la Universidad Católica de Chile con diagnóstico de septicemia en dos períodos separados de dos aáos cada uno: I de julio de 1987 a $\mathbf{3 0}$ de junio de 1989 y I de enero de 1995 a 3 I de diciembre de 1996.

El diagnóstico de septicemia se basó en al menos un bemocultivo positivo en pacientes con síndrome clínico compatible. Se considero que la septicemia era connatal $(0$ precoz) cuando el hemocultivo positivo se obluvo en las primeras 72 horas de vida y se la califico de nosocomial to tardía) si el hemocultivo positivo fue posterior a ellas. La información de los cultivos positivos se obtuvo prospectivamente en este servicio. Los datos restantes se registra. ron en forma retrospectiva e incluyen peso de nacimiento. edad gestacional y sexo, de análisis bacteriologico (germen y sensibilidad) y letalidad. En el segundo período se obtuvo, además, información sobre cultivo de líquido cefalorraquídeo obtenido simultáneamente con el hemocultivo.

En el análisis estadístico se emplé la prueba de $t$ de Student para comparar variables independientes, y la de chi cuadrado o la prueba exacta de Fischer para comparar proporciones. Se fijo como estadísticomente significativo el valor de $\mathrm{p}$ menor a 0,05 .

\section{Resultados}

Durante el primer período se registraron 6227 nacimientos en nuestra maternidad y 5170 en el segundo. En el total de ambos períodos se diagnosticó septicemia neonatal en 63 recién nacidos, 18 en el primer periodo y 45 en el segundo. En el segundo período se registró mayor proporción de septicemias a expensas de las infecciones rosocomiales, pero disminuy 6 la letalidad (tabla l). En las septicemias connatales el germen preponderante fue Streptococcus grupo B sin diferencias entre ambos períodos. Sin embargo, en las septicemias nosocomiales casi se triplicaron los aislamientos de Staphylococcus, emergiendo el $S$. epidermidis coagulasa negativo como un agente importante (tabla 2). En el primer período todos los Staphylococcus aislados eran sensibles a cloxacilina, mientras sólo $33 \%$ lo fueron en el segundo.

La proporción de hemocultivos positivos en el segundo período fue $7,5 \%$. En este mismo período se realizó punción lumbar en $45 \%$ de

\section{Tabla 1}

Incidencia, letalidad y características de 63 recién nacidos con septicemia

\begin{tabular}{|c|c|c|}
\hline & $\begin{array}{c}\text { Periedo I } \\
(7 / 1987-6 / 1989)(1\end{array}$ & $\begin{array}{c}\text { Período } 2 \\
1 / 1995-12 / 1996)\end{array}$ \\
\hline & a: 18 & $\mathrm{D} \div 45$ \\
\hline Incidencia (por mil) & 2,9 & $8.7^{*}$ \\
\hline connalales & 1.6 & 2,5 \\
\hline nosocomiales & 1,3 & $6,2^{*}$ \\
\hline Letalidad $(\%)$ & 44 & $2.2^{*}$ \\
\hline Peso nacieniento $(\mathrm{g} \times \pm \mathrm{DE})$ & $1664 \pm 1101$ & $1900 \pm 1098$ \\
\hline Edad gestacional (sem $x \pm D E$ ) & $30,8 \pm 5$ & $32,5 \pm 5$ \\
\hline Sexo $\mathrm{M} / \mathrm{F}$ (管) & $61 / 39$ & $47 / 53$ \\
\hline
\end{tabular}

${ }^{*} p<0,05$

\section{Tabla 2}

Características bacteriológicas en 63 reciến nacidos con septicemia

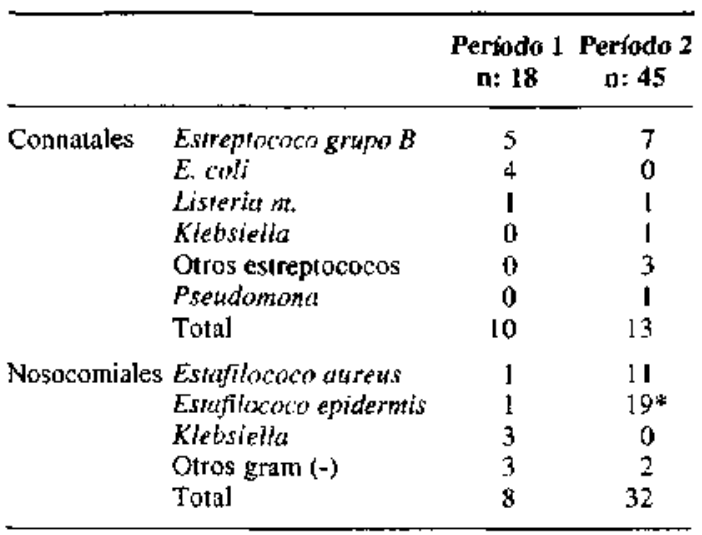

$* 0<0,05$ 
los casos y en todas las muestras los cultivos dieron resultado negativo.

\section{Comentario}

En este estudio se encuentró aumento de la incidencia y disminución de la letalidad de las septicemias neonatales en el período enero 1995 a diciembre 1996, en comparación con el de julio 1987 a junio 1989. La explicación para el aumento en la incidencia está probablemente relacionada a la mayor sobrevida de recién nacidos de alto riesgo. Confirma esta apreciación el que este aumento en la incidencia sea en base a las septicemias nosocomiales, como también ha sido observado en otros lugares ${ }^{6}$. La mejoría en el pronóstico se puede atribuir a una pesquisa y tratamiento más precoces, sumado a los avances en el manejo general de estos pacientes.

La preponderancia actual de los gérmenes gram positivos en la etiología de las infecciones neonatales ha sido comunicada también en otras experiencias $^{2,6,8}$. En las septicemias connatales el Streptococcus grupo B es el agente causal más frecuente con una prevalencia de aproximadamente $50 \%$ en ambos períodos ${ }^{5.6}$. En las septicemias nosocomiales destacan el aumento explosivo de las infecciones estafilocócicas, debido a la emergencia del Staphylococcus epidermidis, y aumento de su resistencia a la cloxacilina, igual que en otros países $\left.{ }^{6,8}, 1\right]-13$. Esta información es muy importante para determinar la combinación antibiótica a emplear cuando se sospecha una septicemia.

La incidencia de meningitis como complicación de las septicemias neonalales parece ir en declinación desde aproximadamente $25 \%$ a 50 $10 \%$ en la actualidad ${ }^{6}, 1+16$. Esta disminución podría ser consecuencia de diagnóstico y terapias de infecciones más precoces. La ausencia de casos de meningitis bacteriana en esta serie coicide con tal tendencia, aun con la limitante de no haber practicado punción lumbar en todos los casos. Por lo demás, existe controversia respecto de efectuar rutinariamente punciones lumbares a recién nacidos asintomáticos con sospecha de infección connatal ${ }^{17,18}$. En otras ocasiones no se efectúa esta punción por la gravedad del caso, o bien no se logra obtener líquido cefalorraquídeo. La baja frecuencia de hemocultivos positivos concuerda con la estrategia de actuar precozmente, aunque la sospecha de infección bacteriana neonatal sea baja.

\section{Agradecimientos}

Los autores agradecen al Sr. Luis Villaroel por su colaboración en el análisis estadístico de este estudio.

\section{Referencias}

1. Stoll BJ: The global impact of neonatal infection. Clin Perinat 1997; 24: 1-21.

2. Tellerias L Oro MA, Lagos E. Méndez del CE: Infecciones bacterianas neonatales: magnilud y aspectos clínicos. Rev Chil Pediatr 1989; 60: 262-266.

3. OHo MA: Infecciones bacterianas neonatales. Rev Chi] Pediatr 1992:63: 17-20.

4. Yancey $M K$, Duff P. Kubilis P, Clark P. Horn Frentzen $B$ : Risk factors for neonatal sepsis. Obstet Gynecol 1996: 87: 188-194.

5. Gladstone M. Ehrenkranz RA. Edberg SC, Baitimor $R S$ : A ten-year review of neonatal sepsis and comparison with the previous fifty-year experience. Pediatr Infect Dis J 1990; 9: 819-825.

6. Philip AGS: The changing face of neonatal infection: Experience ar a regional medical center. Pediatr Infect Dis J 1994: 13; 1098-I102.

7. Bennet $R$, Eriksson $M$. Melen $B$, Zetrerström $R$ : Changes in the incidence and spectrum of neonatal septicemia during a fifteen year period. Acta Paediatr Scand 1985; 74: 687-690.

B. Panrick $C C$ : Coagulase-negative staphylococci: Pathogens with increasing clinical significance. J Pediatr 1990: i16: 497-507.

9. Weisman CLE. Stoll BJ. Cruess DF, et ul; Eariy-onset group B streptococcal sepsis: A current assessment. J Pediatr 1992; I21: 428-433.

10. Stoll BJ. Gordon T. Korones SB, et al: Eariy-onset sepsis in very low birth weight neonates: A report from the National Institute of Child Health and Human development Neonatal Research Network. J Pediatr 1996: 129: $72-80$.

11. Sioll BJ. Gordon $T$, Korones $S B$, et at: Late-onset sepsis in very low birth weight neonates: $A$ report from the Nacional Institute of Child Health and Human Development Neonatal Research Network. J Pediatr 1996: 129: 63-71.

12. Oto MA, Pinto ME, Maritinez V. el al: Control de infecciones por Sraphylococcus aureus resistente a meticilina en una unidad de neonatología. Rev Chil Pediatr 1992; 63; 134-138.

13. Goynes RP, Edwards JR, Jarvis WR, Culver DH. Tolson IS, Martone WJ, and the National Nosocomiat Infections Surveillance Sysiem: Nosocomtal infections among neonates in high-risk nurseries in the United States. Pediatrics 1996; 98: 357-36 I.

14. Bell AH, Brow D. Halliday HL, McClure G. McReid $M$ : Meningitis in the newborn a 14 year review. Arch Dis Child 1989; 64: 873-874. 
15. Schuersenski $d_{1}$ McInfyre L, Buter $C R$; Lumbar puncture frequency and cerebrospinal fluid analysis in the neonate. AJDC 199I; 145: 54-58.

16. Hristeva 1 , Burler $Y$, Booy $R$, King A, Wikinson AR: Value of cerebroespinal fluid examinalion in the diagnosis of meningitis in the newborn. Arch Dis Child 1993; 69: 514-517.
17. Wiswell TE, Baumgart S, Gannon CM, Spitzer AR: No lumbar puncture in the evaluation for early neonalal sepsis: Will meningitis be missed?. Pediatrics 1995; 95: $803-806$.

18. MacMahon P. Jenes, Luavois JD: Routine lumbar punctures in the newborn-are they justified? J Pediatr 1990; 149: 797-799.

\section{AVISO A LOS AUTORES}

Con el objeto de dar prioridad a los trabajos de investigación, en vista de las limitaciones de espacio de la Revista Chilena de Pediatría, el Comité Editorial ha acordado restringir la impresión de casos clínicos a un máximo de dos por cada número. 